\title{
Additional value of linked color imaging in colonoscopy: a retrospective study
}

\section{(ㄷ)(1)}

Authors

Taku Sakamoto ${ }^{1}$, Yutaka Tomizawa ${ }^{1,2}$, Hourin Cho ${ }^{1}$, Hiroyuki Takamaru ${ }^{1}$, Masau Sekiguchi ${ }^{1}$, Masayoshi Yamada ${ }^{1,3}$, Takahisa Matsuda', Yoshitaka Murakami ${ }^{4}$, Yutaka Saito ${ }^{1}$

Institutions

1 Endoscopy Division, National Cancer Center Hospital, Tokyo, Japan

2 Gastroenterology, Harborview Medical Center, Seattle, Washington, United States

3 Division of Molecular Modification and Cancer Biology, National Cancer Center Research Institute, Tokyo, Japan

4 Department of Medical Statistics, Toho University, Tokyo, Japan

submitted 18.2.2019

accepted after revision 25.6.2019

\author{
Bibliography \\ DOI https://doi.org/10.1055/a-0982-2904 I \\ Endoscopy International Open 2019; 07: E1448-E1454 \\ (c) Georg Thieme Verlag KG Stuttgart · New York \\ eISSN 2196-9736
}

Corresponding author

Taku Sakamoto, Endoscopy Division, National Cancer Center Hospital, 5-1-1 Tsukiji, Chuo-ku, Tokyo 104-0045, Japan

Fax: +8135423815

tasakamo@ncc.go.jp

\section{ABSTRACT}

Background and study aims Linked color imaging (LCI), a newly developed optical modality, enhances mucosal surface contrast. We aimed to evaluate the efficacy and feasibility of insertion-phase $\mathrm{LCl}$ in terms of additional benefit of colorectal polyp detection over that obtained with white light imaging (WLI).

Patients and methods We consecutively enrolled eligible patients from November 2017 to June 2018. During colonoscopy, LCl or WLI was alternatively applied on scope insertion and LCl was applied on scope withdrawal. Patients were divided into two groups according to the protocolized difference of imaging modality used in the scope insertion phase ( $\mathrm{LCl}$ and $\mathrm{WLI}$ groups). Group differences in clinical outcomes were evaluated.

Results A total of 138 patients were enrolled in this study, with equal numbers of patients assigned to the $\mathrm{LCI}$ and WLI groups. Most of the lesions located in the proximal colon were detected during the withdrawal phase, without a difference in proportions between the two groups. However, in the $\mathrm{LCl}$ group, eight of 49 lesions (16\%) located in the sigmoid and rectosigmoid colon were only detected during the insertion phase, and no such lesions $(0 \%)$ were detected during the insertion phase in the WLI group ( $P=0.045)$. Conclusions This study showed the efficacy and feasibility of $\mathrm{LCl}$ in improving colorectal polyp detection in the sigmoid colon, especially during insertion. Further studies are warranted to validate the results of our single-center study.

\section{Introduction}

Colonoscopy has been widely accepted as a highly effective tool to decrease mortality of colorectal cancer [1-5]. To best achieve the clinical goal of reduced mortality, all neoplasms should be detected during the examination and treated appropriately $[6,7]$. However, colonoscopy is not flawless in detection of lesions; there is a substantial risk of missing lesions due to anatomical features, preparation quality, or endoscopists' experience [8-11]. Moreover, the post-polypectomy surveillance interval is set by findings from the initial colonoscopy, including the number, size, and histological findings of detected lesions [6,12]. Therefore, it is crucial to detect all neoplastic lesions to determine appropriate long-term management and achieve the ultimate purpose of colon cancer screening.

Several anatomical features contribute to risk of missed lesions. Deep haustral folds and sharp curves are associated with missed lesions. Haustral folds are usually deeper in the proximal than in the distal colon; this is considered to be one of the common causes for missed lesions in the proximal colon. On the other hand, the sigmoid colon is dangling and able to both expand and shrink, like a bellows. Therefore, the sigmoid colon sometimes shows deep folds, and this situation is often seen during the withdrawal phase of colonoscopy. In general, inser- 
tion to the cecum should be completed as quickly as possible, and the search for lesions should be conducted during withdrawal [13]. However, deep folds, which can be encountered in the sigmoid colon in the withdrawal phase, make it difficult to detect subtle neoplastic lesions, especially behind the folds. Hence, searching for lesions during insertion is also important for decreasing risk of missed lesions, especially in the sigmoid colon.

Linked color imaging ( $\mathrm{LCl}$ ), a newly developed optical technology, can enhance mucosal surface contrast as compared to that with white light imaging (WLI) due to its optical characteristics [14-16]. With $\mathrm{LCI}$, extensive observation of the colonic lumen can be obtained and polyp lesions can be detected on the distant view. Thus, $\mathrm{LCl}$ might contribute to detection of a greater number of sigmoid colon polyp lesions during insertion compared to that obtained with WLI.

The aim of the current study was to evaluate efficacy and feasibility of insertion-phase $\mathrm{LCl}$ in terms of the additional benefit of colorectal polyp detection over that obtained with WLI.

\section{Patients and methods}

\section{Study design}

This study investigated endoscopic outcomes by retrospective review of medical records.

\section{Patients}

Patients who underwent colonoscopy performed by a single, experienced endoscopist at the National Cancer Center Hospital in Tokyo, Japan between November 2017 and June 2018 were considered as candidates for this retrospective study. Patients who were referred for age- and risk-appropriate screening colonoscopy, post-polypectomy surveillance, or endoscopic evaluation of a positive fecal occult blood test were enrolled. Patients with inflammatory bowel disease, polyposis syndrome, and postsurgical colorectal cancer surveillance were excluded.

\section{Endoscopic procedure}

Following bowel preparation, nurses assessed bowel cleansing and permitted colonoscopy only after achievement of a sufficiently clean state. Quality of bowel preparation during colonoscopy was scored according to the extent of mucosal visualization after suctioning fluid residue, in accordance with the Aronchick Bowel Preparation Scale: excellent ( $\geq 95 \%$ mucosal visualization), good (90\%-95\% mucosal visualization), fair ( $80 \%$ - $90 \%$ mucosal visualization), and poor (<80\% mucosal visualization).

Colonoscopy was performed using an endoscope system (LL4450; Fujifilm Co, Tokyo, Japan) and high-definition colonoscope (EC-600ZP, EC-600ZP7; Fujifilm Co, Tokyo, Japan). Use of $\mathrm{LCl}$ or WLI during colonoscope insertion, which is not regulated by the Japanese Pharmaceutical Affairs Law, alternated between consecutive patients, at the endoscopist's discretion, during the study period. LCl was performed during withdrawal in all patients. All lesions were photographed immediately after detection, during both insertion and withdrawal. We did not resect any lesions during insertion. Detailed observation, with magnifying blue laser imaging (BLI) or chromoendoscopy, was performed only during withdrawal, if necessary. Lesions diagnosed as an adenoma, high-grade intramucosal neoplasm, or sessile serrated lesion (SSL) were endoscopically removed. The resect-and-discard strategy was allowed for lesions smaller than $5 \mathrm{~mm}$ when it was difficult to retrieve the resected specimen. Hyperplastic polyps smaller than $10 \mathrm{~mm}$ in the sigmoid to rectosigmoid colon were excluded from analysis. Insertion and pure observation time were recorded for all cases, and time of endoscopic resection was removed from the later measure.

For determination of identical lesions detected during insertion and withdrawal, we evaluated lesion size, macroscopic type, and color and number of lesions in the sigmoid colon by using still images. Location was determined from the splenic flexure finding.

\section{Outcomes}

The primary endpoint was proportion of lesions in the sigmoid colon only detected during insertion. Secondary endpoints were mean number of lesions detected in the sigmoid colon per patient. We also defined SSLs as sessile serrated adenomas/polyps (SSA/Ps) and traditional serrated adenomas (TSAs). Adenomas were defined as an adenomatous lesion, with the exception of tubulovillous adenomas (TVAs); TVAs were considered as high-grade intramucosal neoplasms (HGINs) in this study. Index lesions were defined as follows: adenomas $\geq 10 \mathrm{~mm}$, having villous histology; HGINs; cancer; SSLs $\geq 10 \mathrm{~mm}$; or serrated lesions harbouring dysplasia, including TSAs.

\section{Statistical analysis}

Continuous data are reported as means and $95 \%$ confidence intervals (Cls); categorical data are reported as numbers, rates, and $95 \% \mathrm{Cls}$. Differences in means between $\mathrm{LCl}$ and WLI groups were evaluated using mean differences with $95 \% \mathrm{Cls}$. Differences in distribution between $\mathrm{LCl}$ and WLI groups were evaluated using the Chi-square test. In addition, proportional odds models were used to explore the relationship between characteristics of detected lesions and group assignment. In this model, the generalized estimating equation (GEE) method was applied to account for correlated observations among lesions. Odds ratios for both the GEE and independent models are reported. Proportional odds models with GEE were performed using SAS release 9.40 software (Cary, North Carolina, United States). Other statistical analyses were performed using EZR software (http://www.jichi.ac.jp/saitama-sct/SaitamaHP.files/statmed. html) [17].

\section{Ethics}

The study was conducted according to guidelines of our Institutional Review Board, which approved this study and waived the requirement for informed consent. Patients were able to opt out of participation on our hospital website. All patients provided written informed consent for undergoing the colonoscopic and endoscopic treatments. 
- Table 1 Clinical characteristics according to group.

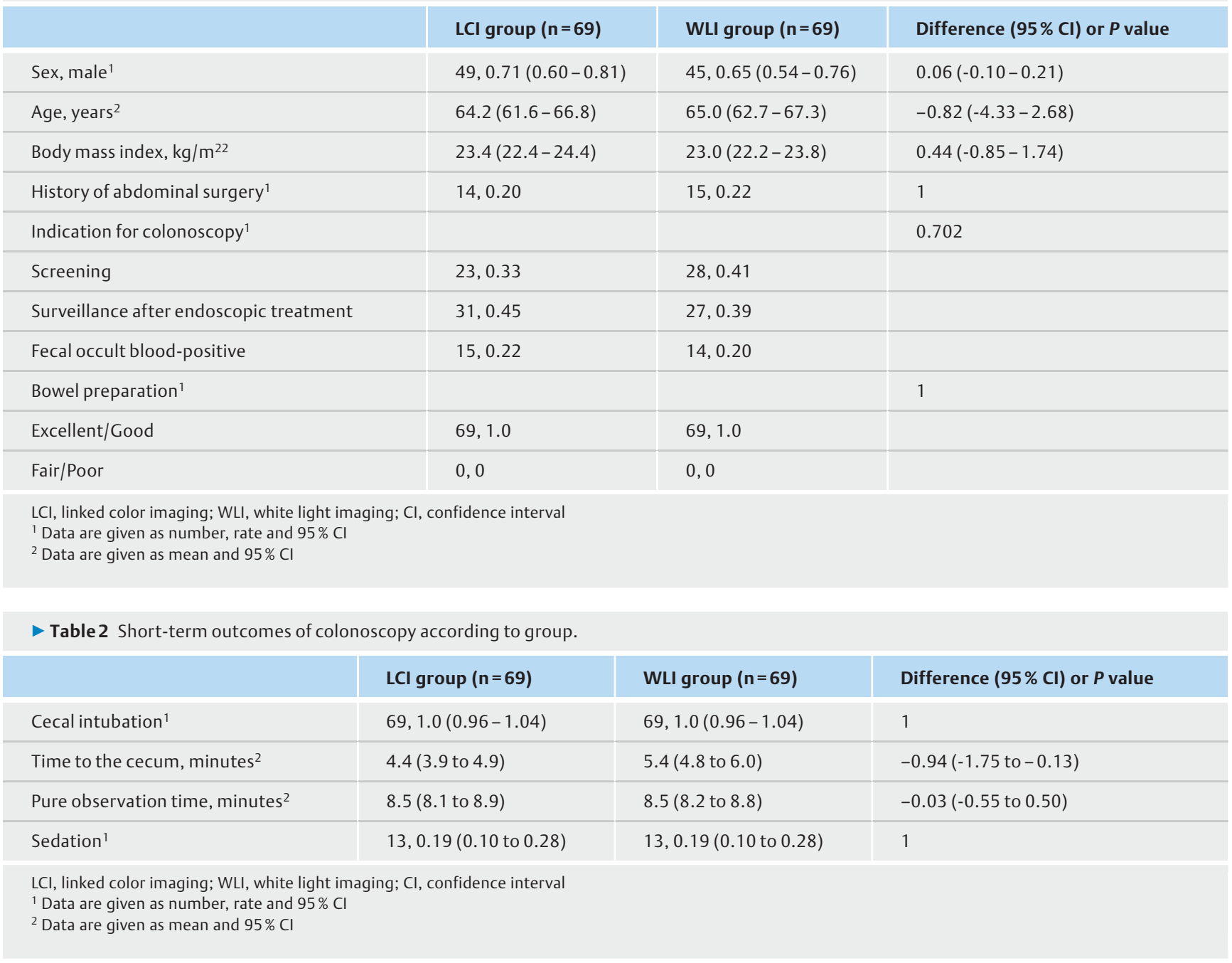

\section{Results}

A total of 138 patients were consecutively enrolled, with equal numbers of patients assigned to the $\mathrm{LCl}$ and WLI groups. Clinical characteristics and short-term outcomes of the colonoscopy are shown, according to group, in $\downarrow$ Table 1 and $>$ Table 2 . Baseline patient characteristics of sex, age, body mass index, history of abdominal surgery, indication for colonoscopy, and bowel preparation assessment during examination did not significantly differ between the two groups. Among colonoscopic outcomes, only insertion time statistically differed between the two groups, with a shorter insertion time (by 1 minute) in the $\mathrm{LCl}$ group than in the WLI group.

In the $\mathrm{LCl}$ group, 144 lesions were detected in 69 patients. Of these, 134 lesions were resected using cold snare polypectomy (CSP) or cold biopsy (BP); eight lesions were resected by hot snare polypectomy (PO), endoscopic mucosal resection (EMR), or endoscopic submucosal dissection; BP was used to attempt estimation of histology for one submucosal tumor (SMT); and one lesion was treated by surgery due to a diagnosis of deep submucosal invasion. In the WLI group, 132 lesions were de- tected in 69 patients. Of these, 127 lesions were resected by CSP or BP, and five lesions were treated by PO or EMR. Furthermore, 20 and seven lesions were discarded from the $\mathrm{LCI}$ and WLI groups, respectively, after CSP due to size criteria. Procedural and histological characteristics of lesions detected are shown in $>$ Table 3 . In the independent odds models, lesion size $(P=0.958)$, histology $(P=0.303)$, and endoscopic diagnosis $(P=0.914)$ were not significantly different between the two groups. However, location $(P=0.027)$ and morphology $(P=$ 0.048 ) were significantly different between the groups. In the proportional odds models with GEE, which accounted for correlated observations, morphology was marginally significant, and location was the only factor that significantly differed between the groups. Odds ratios for both the GEE and independent models are shown in $>$ Table 3 . In both groups, approximately $70 \%$ of lesions were categorized as diminutive $(<5$ $\mathrm{mm})$, and approximately $20 \%$ of lesions were small (6-9 mm). Regarding endoscopic diagnoses, in both groups, approximately $80 \%$ of lesions were estimated as adenomas, fewer than $10 \%$ were estimated as SSLs, and $2 \%$ to $3 \%$ were estimated as HGINs or cancer. Furthermore, $16(11 \%)$ and 10 (8\%) lesions were ca- 

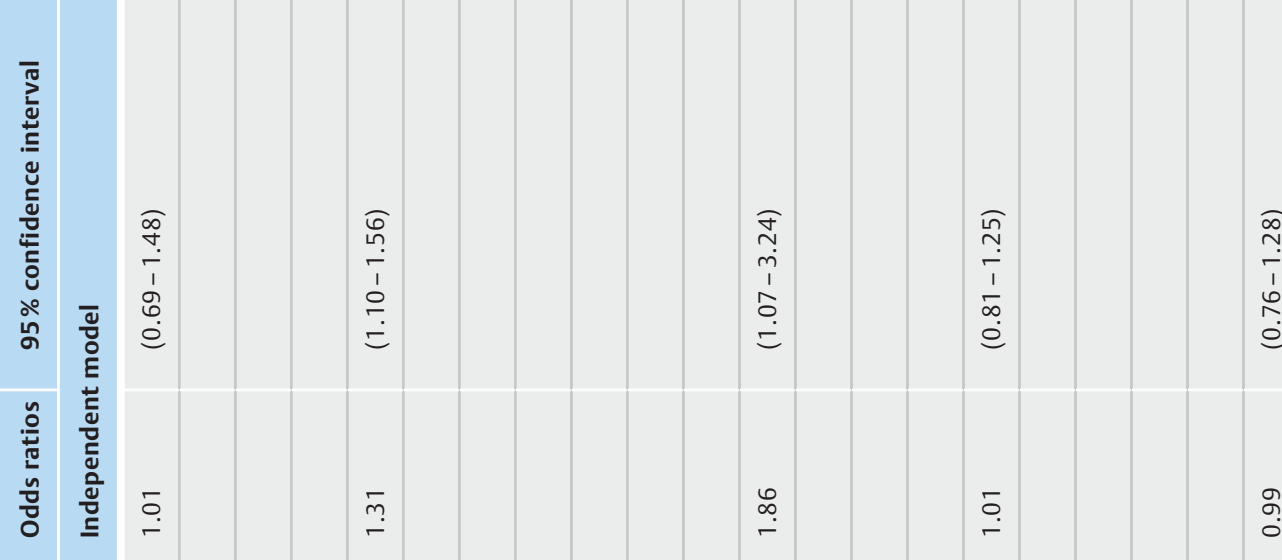

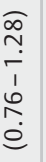

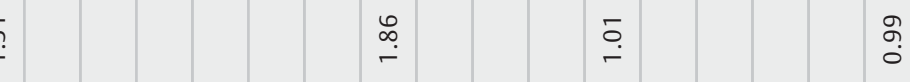

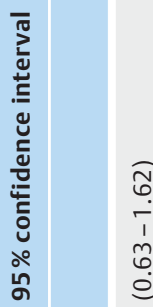

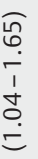

$\widehat{a}$
$\infty$
$p$
$p$
$\vdots$
$o$
$o$
0

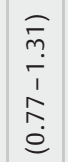

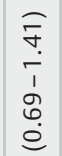

空蒙

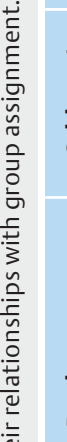

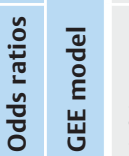

$\stackrel{\bar{m}}{r}$

$\stackrel{\infty}{\infty}$

$\stackrel{-}{i}$

g.

$\sum_{n=0}^{5}+$

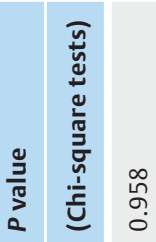

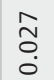

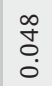

$\stackrel{\substack{m \\ m \\ 0}}{n}$

它

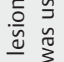

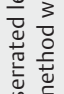

畜嵌

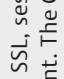

E

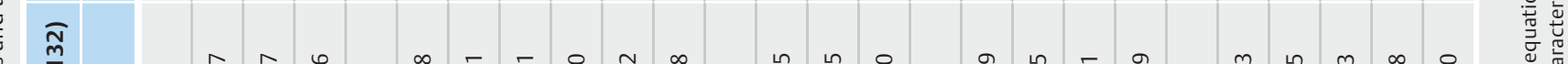

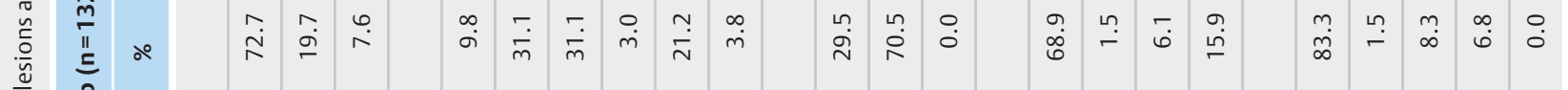

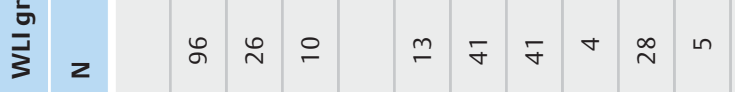

ウి

至焉

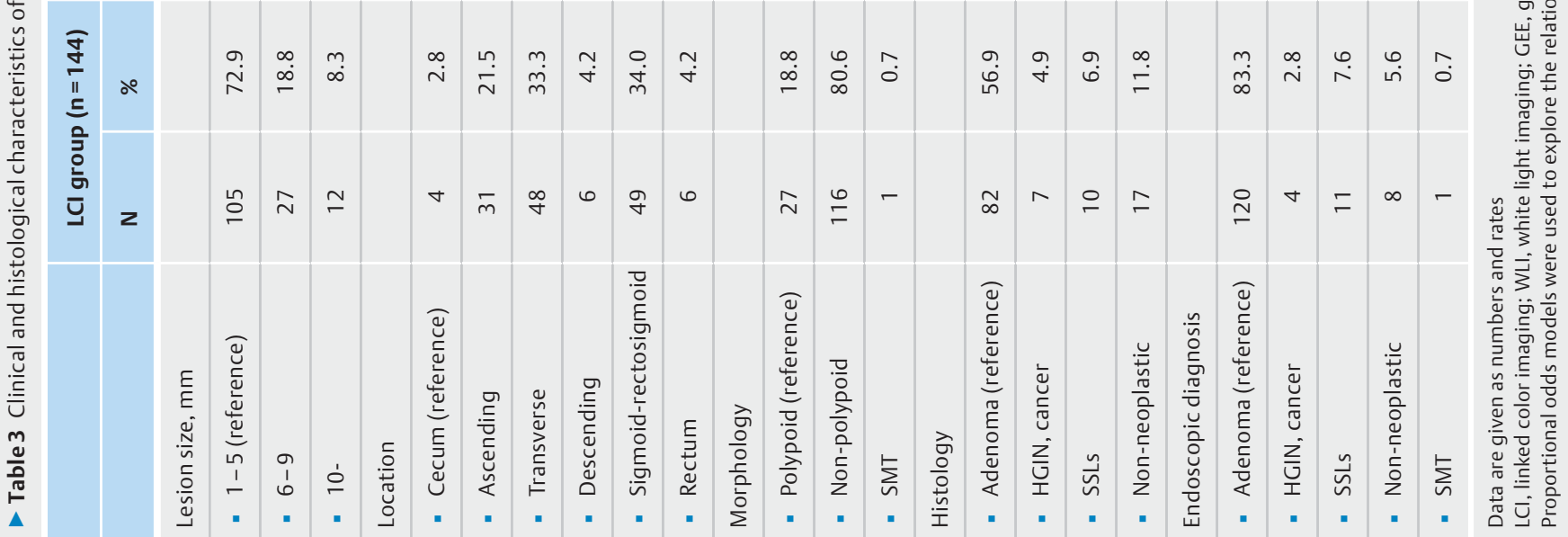




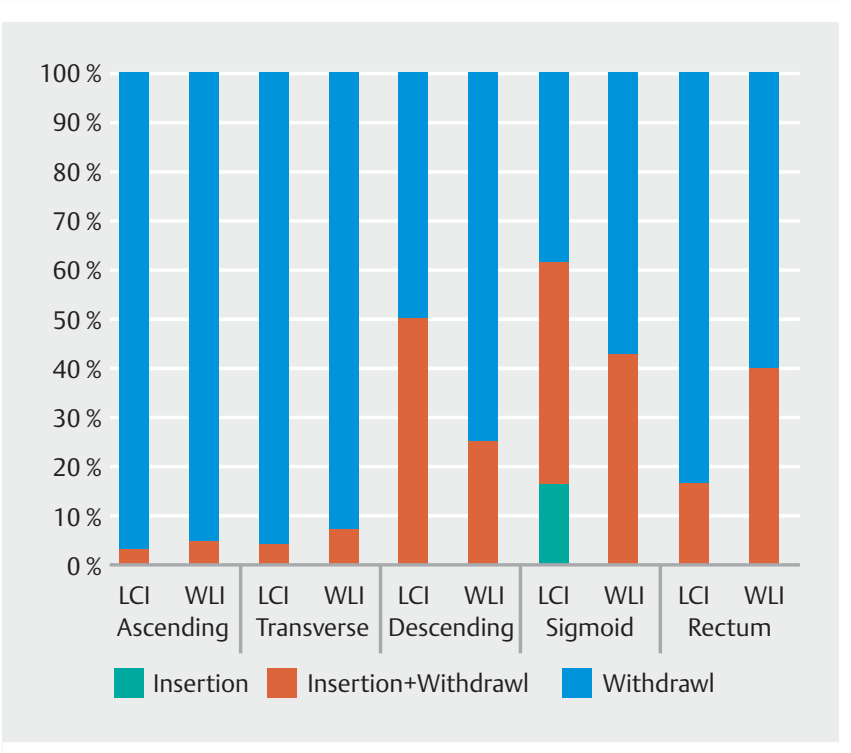

- Fig. 1 Relationship between location of detected lesion and phase in which lesion was detected. $\mathrm{LCl}$, linked colour imaging; WLI, white light imaging

tegorized as index lesions in the $\mathrm{LCl}$ and WLI groups, respectively.

Most lesions located in proximal colon were detected during withdrawal, with no significant difference in proportion between the $\mathrm{LCl}$ and WLI groups ( $\triangleright$ Fig. 1). It is of note that lesions located in the sigmoid and rectosigmoid colon showed a different pattern of results. Specifically, in the LCI group, eight of 49 lesions (16\%) could only be detected during insertion, and no such lesions ( $0 \%)$ were detected during insertion in the WLI group $(P=0.045)$. In these cases, we reinserted the colonoscope back to the sigmoid colon; seven of eight lesions could be re-detected. Only one lesion which was diagnosed as a diminutive adenoma could not be detected again. - Fig. 2 shows a representative case in which we were able to detect the lesion only during insertion.

\section{Discussion}

Results of the current study suggest that LCI improves detection of colorectal polyp lesions during insertion phase, especially in the sigmoid colon.

Detection of colorectal lesions is generally attempted during withdrawal, and insertion phase is usually devoted to reaching the cecum as quickly and safely as possible. Therefore, appropriate colorectal polyp detection has been evaluated during withdrawal. There are two published randomized controlled studies on efficacy of $\mathrm{LCl}$ for detection of colorectal lesions: Min at el. [18] reported its efficacy in a randomized crossover trial, and Paggi et al. [19] reported its efficacy in a randomized tandem colonoscopy study. The former study demonstrated that $\mathrm{LCl}$ improved detection of not only polyps but also adenomas, and the latter study demonstrated that $\mathrm{LCl}$ reduced the possibility of missing a lesion in the right colon. Both studies appear to have evaluated the number of polyps detected during withdrawal phase.

We have experienced cases in which we could not re-find polyps located in the sigmoid colon that were initially detected during insertion. Therefore, we intended to evaluate efficacy of $\mathrm{LCl}$ during insertion phase for detection of colorectal lesions located in the sigmoid colon. We found that approximately $10 \%$ of lesions in the sigmoid colon could only be detected during insertion. Moreover, this superior detectability during the insertion phase was demonstrated in the $\mathrm{LCl}$ group but not in the WLI group. This result suggests the clinical utility of $\mathrm{LCl}$ in addition to conventional colonoscopy. It is of note that insertion time was shorter in the LCl group than in the WLI group; however, the actual difference was 1 minute. From these results, applying $\mathrm{LCl}$ during insertion might be recommended. One potential issue with $\mathrm{LCl}$ is that halation due to its strong brightness conversely worsens the visibility of the lumen, and some endoscopists might feel discomfort due to a lack of experience. However, one can immediately and easily change the light mode from LCI to WLI with the push of a single button.

In this study, we could not prove the efficacy of $\mathrm{LCl}$ for advanced polyps, detection of which is also considered to be important for prevention colorectal cancer mortality. In the current study, most of the detected lesions were diagnosed as tubular adenomas smaller than $5 \mathrm{~mm}$, and the overall percentage of advanced polyps was $10 \%$. Several factors may explain this result. Given the indications for the current study, more than half the patients were likely categorized as average risk for colorectal cancer. In addition, the patients who were undergoing surveillance after endoscopic treatment should have already had most of their advanced polyps resected on prior colonoscopy within the past 5 years. It is conceivable that advanced polyps larger than $10 \mathrm{~mm}$ would be detected more easily than diminutive polyps, and it is difficult to prove the additional benefit of $\mathrm{LCl}$ to currently available imaging modalities for detection of such advanced neoplastic polyps. The current study was intended to evaluate LCl's overall detection ability. We think the ability to detect diminutive polyps, which are more difficult to detect than larger lesions, should more precisely determine use of the technology.

The current study results demonstrated that more attention should be paid to the features of missed lesions located in the sigmoid colon. Most of the lesions located in the right colon could be detected during withdrawal. In contrast, fewer than $30 \%$ of lesions in the WLI group could be recognized during both insertion and withdrawal, and fewer than $70 \%$ of lesions in the $\mathrm{LCl}$ group could be recognized during both insertion and withdrawal. Moreover, approximately $10 \%$ of lesions could only be detected during insertion. This result might reflect a unique feature of sigmoid colon lesions. In general, one of the causes for missed lesions is thought to be existence of a blind spot due to a sharp curvature or deep fold, and the right colon is the part of the colon where we often encounter missed lesions. Generally, the easiest way to overcome this issue is to perform a detailed observation using retroflexion. However, retroflexion is difficult in the sigmoid colon, as it easily expands and contracts. Hence, detection during both insertion and withdrawal 

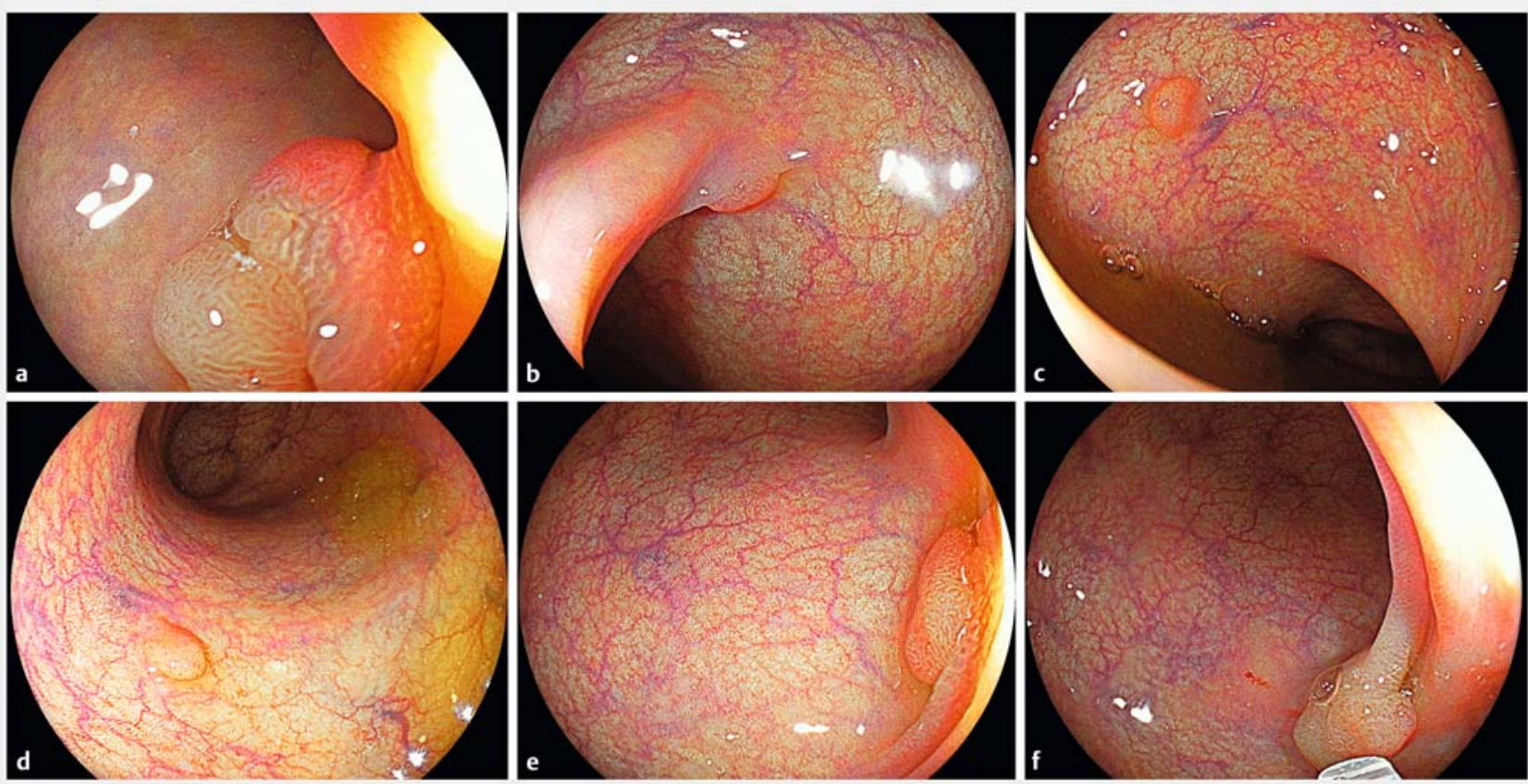

- Fig. 2 a representative case showing a lesion missed during the withdrawal phase. a, b, $\mathbf{c}$ Three lesions were detected in order during the insertion phase. c, d, e Only two lesions were detected during the withdrawal phase. The lesions in a and $\mathbf{e}$, and $\mathbf{c}$ and $\mathbf{d}$ were judged to reflect the same lesion by comparing the size and morphology. The lesion in $\mathbf{b}$ could not be identified during withdrawal but was detected by reinsertion after observation of the rectum.

is thought to be crucial to decrease incidence of missed lesions in the sigmoid colon.

The current study has some limitations. First, it was not designed as a prospective randomized study, and was conducted at a single institution, with a limited number of cases. Namely, it is impossible to exclude the effect of confounding biases in the comparisons of outcomes. Thus, a larger-scale prospective study should be conducted to validate the results of this observational study. The current results can be used to determine the appropriate sample size for a future trial. Second, perfect identification of lesions detected during both insertion and withdrawal, or in just one phase, was difficult in this study. However, marking lesions for resection during insertion could further aid in identifying lesions detected in both or one phase. Moreover, such methodology is not recognized as general procedure, but we consider that the current method of identifying lesions detected in both/one phase is the currently the best possible manner, even though it was not perfect. Third, the current study results are based on clinical outcomes of a colonoscopy performed by an experienced endoscopist. Efficacy of insertion-phase $\mathrm{LCl}$ should be confirmed in a larger number of endoscopists, ideally including endoscopists with variable expertise. We plan to overcome these issues in a future validity study. Fourth, it was difficult to evaluate efficacy of insertionphase $\mathrm{LCl}$ for detection of advanced adenomas because of their low prevalence in the study population. Thus, patients categorized as having a higher risk of cancer should have been enrolled. However, the main goal of this study was to demonstrate superior lesion detectability of $\mathrm{LCl}$ in comparison to that achieved with WLI. Therefore, diminutive lesions, which are more difficult to detect than large polyps, should be an appropriate study object to prove lesion detection ability. Moreover, we believe that lesion detection during insertion is clinically very important, decreasing risk of a missed lesion located in the sigmoid colon; however, there is paucity of supporting literature. Further research is needed to provide sufficient evidence of the importance of insertion-phase imaging during routine colonoscopy.

\section{Conclusion}

In conclusion, this study showed that use of $\mathrm{LCl}$ during the insertion phase of colonoscopy contributes to better detection of lesions in the sigmoid colon than that achieved with conventional colonoscopy with WLI. In addition, there are some lesions that can only be detected during insertion, especially in sigmoid colon, due to its anatomical features and practical clinical features.

\section{Competing interests}

None 


\section{References}

[1] Winawer S], Zauber AG, Ho MN et al. Prevention of colorectal cancer by colonoscopic polypectomy. The National Polyp Study Workgroup. N Engl J Med 1993; 329: 1977 -1981

[2] Citarda F, Tomaselli G, Capocaccia R et al. Efficacy in standard clinical practice of colonoscopic polypectomy in reducing colorectal cancer incidence. Gut 2001; 48: 812-815

[3] Singh H, Nugent Z, Demers AA et al. The reduction in colorectal cancer mortality after colonoscopy varies by site of the cancer. Gastroenterology 2010; 139: 1128-1137

[4] Niikura R, Hirata Y, Suzuki N et al. Colonoscopy reduces colorectal cancer mortality: A multicenter,long-term, colonoscopy-based cohort study. PLoS One 2017; 12: e0185294

[5] Kahi C], Pohl H, Myers L] et al. Colonoscopy and colorectal cancer mortality in the Veterans Affairs Health Care System: a case-control study. Ann Intern Med 2018; 168: 481 - 488

[6] Hassan C, Quintero E, Dumonceau JM et al. Post-polypectomy colonoscopy surveillance: European Society of Gastrointestinal Endoscopy (ESGE) Guideline. Endoscopy 2013; 45: 842 - 851

[7] Pohl H, Robertson DJ. Colorectal cancers detected after colonoscopy frequently result from missed lesions. Clin Gastroenterol Hepatol 2010; 8: 858-864

[8] Pickhardt PJ, Nugent PA, Mysliwiec PA et al. Location of adenomas missed by optical colonoscopy. Ann Intern Med 2004; 141: 352 - 359

[9] Laiyemo AO, Doubeni C, Sanderson AK 2nd et al. Likelihood of missed and recurrent adenomas in the proximal versus the distal colon. Gastrointest Endosc 2011; 74: 253-261

[10] Samadder NJ, Curtin K, Tuohy TM et al. Characteristics of missed or interval colorectal cancer and patient survival: a population-based study. Gastroenterology 2014; 146: 950 - 960
[11] Pickhardt PJ, Nugent PA, Mysliwiec PA et al. Location of adenomas missed by optical colonoscopy. Ann Intern Med 2004; 141: 352 - 359

[12] Lieberman DA, Rex DK, Winawer S] et al. Guidelines for colonoscopy surveillance after screening and polypectomy: a consensus update by the US Multi- Society Task Force on Colorectal Cancer. Gastroenterology 2012; 143: $844-857$

[13] Rex DK. Endoscopy: insertion versus withdrawal phases for polyp detection. Nat Rev Gastroenterol Hepatol 2009; 6: 443 - 444

[14] Sun X, Dong T, Bi Y et al. Linked color imaging application for improving the endoscopic diagnosis accuracy: a pilot study. Sci Rep 2016; 6: 33473

[15] Suzuki T, Hara T, Kitagawa Y et al. Magnified endoscopic observation of early colorectal cancer by linked color imaging with crystal violet staining (with video). Gastrointest Endosc 2016; 84: 726-729

[16] Yoshida N, Naito Y, Yasuda R et al. Linked color imaging improves the visibility of various featured colorectal polyps in an endoscopist's visibility and color difference value. Int J Colorectal Dis 2017; 32: 1253 1260

[17] Kanda Y. Investigation of the freely available easy-to-use software 'EZR' for medical statistics. Bone Marrow Transplant 2013; 48: 452 458

[18] Min M, Deng P, Zhang W et al. Comparison of linked color imaging and white-light colonoscopy for detection of colorectal polyps: a multicenter, randomized, crossover trial. Gastrointest Endosc 2017; 86: $724-730$

[19] Paggi S, Mogavero G, Amato A et al. Linked color imaging reduces the miss rate of neoplastic lesions in the right colon: a randomized tandem colonoscopy study. Endoscopy 2018; 50: 396-402 